\title{
Application of image analysis to the prediction of EBC barley kernel weight distribution
}

\author{
António L. Amaral a,b,*, Orlando Rocha ${ }^{\mathrm{a}}$, Cristina Gonçalves ${ }^{\mathrm{c}}$, António A. Ferreira ${ }^{\mathrm{c}}$, Eugénio C. Ferreira ${ }^{\mathrm{a}}$ \\ a IBB-Institute for Biotechnology and Bioengineering, Centre of Biological Engineering, Universidade do Minho, Campus de Gualtar 4710-057, Braga, Portugal \\ b Instituto Superior de Engenharia de Coimbra, Rua Pedro Nunes - Quinta da Nora, 3030-199 Coimbra, Portugal \\ c UNICER, Via Norte - Leça do Balio, Apartado 1044, 4466-955 S. Mamede de Infesta, Portugal
}

\section{A R T I C L E I N F O}

\section{Article history:}

Received 30 April 2009

Received in revised form 26 June 2009

Accepted 5 July 2009

\section{Keywords:}

Barley analysis

Kernel size

Image analysis

Partial Least Squares

\begin{abstract}
A B S T R A C T
It is known that the barley kernel size is an important factor regarding the uniformity of the malting and brewery processes, barley valuation, approval and market value. In order to facilitate the barley purchasing process, a fast field technique for kernel size evaluation, such as the image analysis technique proposed in this work, would be greatly appreciated as a fast and simple procedure for barley selection. In this study a close correlation between the image analysis and the standard EBC was obtained with a correlation factor of 0.999 and a regression coefficient of 0.991 between the two methodologies. The proposed IA methodology was found to accurately predict the Scarlett and Prestige barley varieties weight distribution especially when considering the crucial 'business transactions selection' classes.
\end{abstract}

(c) 2009 Elsevier B.V. All rights reserved.

\section{Introduction}

In the process of kernel selection for malt producers or the brewery industry, the evaluation of the kernel size of barley is regarded as the most important step. In fact, the market value of barley depends on the kernel size for valuation and approval of the raw material since it guarantees uniformity of the malting process. In order to facilitate the barley crop purchasing process on the field, an image analysis technique is proposed with the purpose of rapid kernel evaluation. This field technique for kernel size evaluation would then allow for a fast and simple procedure for barley assessment and selection (Amaral et al., 2005).

Currently, image analysis is a well-established complement of grain morphology characterization. The image analysis technique allows the enhancement of images, as well as the identification and automatic isolation of particles for further study. In addition, it is a rapid and time-saving technique that allows for the acquisition of quantitative data that could be very difficult or even impossible to obtain otherwise (Gonzalez and Woods, 1992; Dougherty, 1994; Russ, 1995).

\footnotetext{
* Corresponding author at: Instituto Superior de Engenharia de Coimbra, Rua Pedro Nunes - Quinta da Nora, 3030-199 Coimbra, Portugal. Tel.: +351 239790200. E-mail addresses: Ipamaral@isec.pt (A.L. Amaral), orocha@deb.uminho.pt (O. Rocha), antonio.ferreira@unicer.pt (A.A. Ferreira), ecferreira@deb.uminho.pt (E.C. Ferreira).
}

Recent researches on the inspection of cereal kernels by image analysis, computer vision or microscopic techniques have been reported (Brennan et al., 1996; Andersson et al., 1999; Edney et al., 2002; Brosnan and Sun, 2004). A large number of these studies used image analysis techniques for the classification and identification of cereal varieties or kernel quality (Garciǐa del Moral et al., 1998; Majumdar and Jayass, 1999; Utku, 2000; Shouche et al., 2001; Nielsen, 2003). For instance, Nielsen (2003) proposed a method using Graincheck (Foss Electric AS Hillerød, Denmark) and Perten Single Kernel Characterization System 4100 (Perten Instruments Inc., USA) to evaluate malting barley quality of 25 barley varieties by image analysis, Principal Component Analysis (PCA), and multivariate Partial Least Squares (PLS). Reasonable PLS regression models were obtained for the structural and physical part of the malting quality and established the hardness of the barley kernels as the most important variable in malting performance. In addition, Garciřa del Moral et al. (1998) studied embryo size and kernel volume by image analysis in order to evaluate the malting quality of barley kernels. The results showed the nitrogen content as a good predictor of grain protein content, embryo size and grain volume, and the grain protein content as the only practical predictor of malt extract.

The main objective of the current study was the development of a fast image analysis (IA) methodology to determine barley kernel weight distribution throughout commonly used classes within business transactions for kernel assessment and selection on the field. For that purpose an image analysis software routine was developed in a public domain and open source platform. A first study was then performed for determining the camera min- 


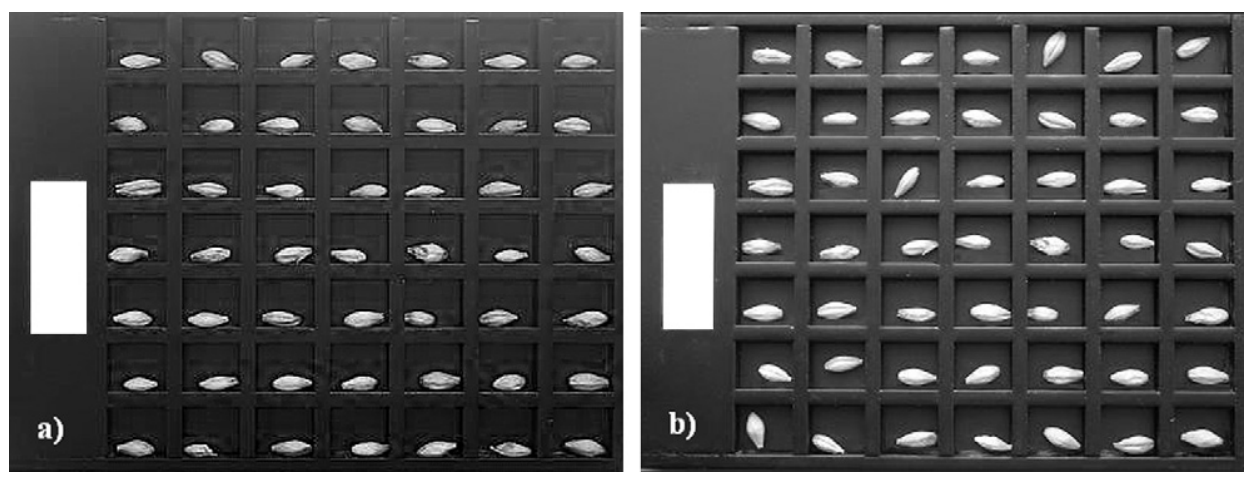

Fig. 1. Images from the side (a) and front (b) views from the kernels.

imum acceptable resolution and the minimum number of kernels that should be acquired. Subsequently, a multivariate Partial Least Squares (PLS) analysis was performed on the IA morphological data, for the determination of the kernel weight and weight distribution, based on the obtained image analysis parameters. In order to evaluate the performance of the proposed image analysis methodology the IA weight distribution was plotted against the standard European Brewery Convention (EBC) weight distribution (Analytica EBC, 1998).

\section{Materials and methods}

The current research was developed in five distinct phases: sampling, kernel size and weight determination by the standard EBC method; development of the image analysis methodology; camera resolution and sampling kernel size dependence; development of the PLS model; and validation of the PLS model.

In the first phase, three different sets of the Scarlett, Nevada, Esterel and Prestige barley varieties were sampled and characterized by Unicer technicians according to the 3.11.1 Analytica EBC "Sieving Test for Barley" method (Analytica EBC, 1998). The kernel size classes studied in this research were the following: less than $2.2 \mathrm{~mm}, 2.2-2.5 \mathrm{~mm}, 2.5-2.8 \mathrm{~mm}$, and greater than $2.8 \mathrm{~mm}$ in minor axis length. Also the greater than $2.5 \mathrm{~mm}$ class (sum of the 2.5-2.8 $\mathrm{mm}$ and greater than $2.8 \mathrm{~mm}$ classes) was studied as it is one of the most important parameters for the commercial assessment and selection of kernels.

The second phase consisted of the development of the image processing and analysis software routine and determination of the morphological parameters of barley. Regarding the image acquisition, emphasis was taken on designing a low cost solution and, therefore, a Hewlett Packard digital camera (2.048 megapixels) was used alongside the public domain Image ( $\mathrm{NIH}$, USA) software. In each image acquisition about 49 kernels were placed in a specially built sampling box containing a $1 \mathrm{~cm}$ ceramics gauge block (Mitutoyo) for kernel size calibration. An average acquisition of 12 images per barley variety was carried out for both front and side views resulting in the characterization of around 600 individual barley kernels. The front view reflects the point of view directly above the barley resting on a horizontal surface, whereas the side view reflects the point of view parallel to the horizontal surface. Images were taken in JPEG 24 bit format ( 8 bit per color channel) at a resolution of $1600 \times 1280$ pixels. Examples of front and side views images are presented in Fig. 1.

Concerning the image processing and analysis program, a set of commands in ImageJ native macro language was developed consisting in five major sections: Identification and measurement of the ceramics gauge block; image pre-processing; image segmentation with barley kernels identification; debris elimination; and finally the morphological parameters determination of barley. Each of these sections is briefly explained below, and the corresponding image processing flowchart is presented in Fig. 2.

Identification and measurement of the ceramics gauge block: After image acquisition the first step of the program consists on the ceramics gauge block size determination and final image calibration by means of the gauge block segmentation either by automatic segmentation by Otsu filter (Otsu, 1979) or manual threshold selection. A careful recognition of the gauge block boundaries by the inspection of the result boundaries image was performed (Fig. 3). This allows the conversion of the morphological parameters of the barley into the proper metric units $(\mathrm{mm})$ : the pixel per $\mathrm{mm}$ ratio was about 130 pixels per $1 \mathrm{~mm}$.

Image pre-processing: This step comprises the conversion of the three RGB color channels ( $\mathrm{R}$-red, G-green and B-blue) image in a single grayscale image and the correction of the non-uniform background illumination. The channel ( $R, G$ or $B$ ) which presents the largest difference between the mean grayscale values of the barley kernels and the background was used as the working image of the barley. In addition, the channel presenting the smallest difference between these two values was used to extract the background working image. Both the working and the background images are determined to each single image given the non-uniformity of the images brightness acquired on the field. Therefore, after preliminary kernel identification in the working image of the barley, the kernels mask was used to blend them (substituted by the median kernel scale value) to the background in the background working image. Then, the background was removed from the working image of the barley by means of a pixel to pixel subtraction of the two images.

Image segmentation with the identification of barley kernels: The third step of the program covers the identification of each of the barley kernels by the prior determination of the kernels containing cells boundaries and seemingly the individual barley kernel segmentation. The segmentation step can be achieved by either automatic means, such as the minimal entropy, histogram inspection (Russ, 1995), or Otsu filter (Otsu, 1979) segmentation methods, or manual threshold selection. The pre-defined threshold method is the histogram inspection, and careful examination of each barley kernel boundary's recognition by the inspection of the result boundaries image (see Fig. 3b) was performed. Only for cases where the resulting binary images did not comply with the barley kernels the use of other threshold selection methods was applied.

Debris elimination: Small debris may be attached to the kernels due to germination tubes, small residues, light reflection effects or close proximity to the boundaries of the cells. Therefore, the elimination of all of the above debris is of critical importance to the barley kernel determination as it raises problems in the minor axis accurate determination. A size-based procedure is enough to eliminate non-attached debris present in the image. However, for attached debris, a more complex procedure is put in practice. Therefore, a 


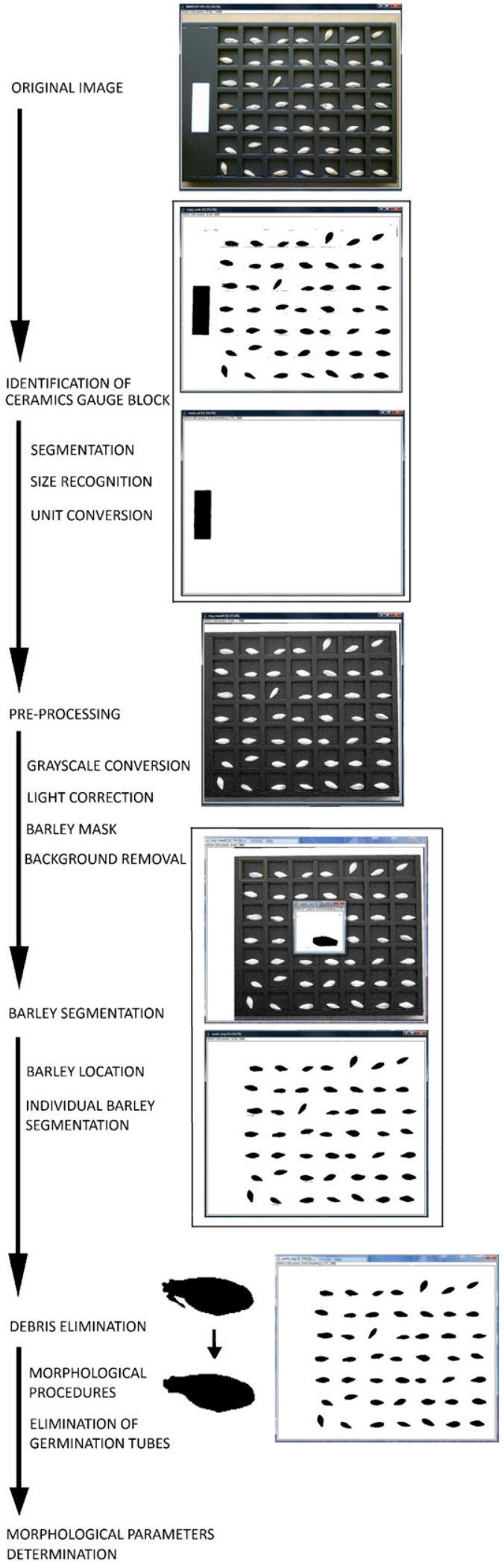

Fig. 2. Image processing flowchart.

methodology based on a series of morphological opening procedures was used in order to recognize and cut off all of the attached debris.

Morphological parameters determination of the barley: In this step both front and side view binary images were treated in order to obtain a detailed description of the overall morphology. For each individual kernel the obtained size parameters were the kernel Height (axial minor axis) and Minimum Feret Diameter (minimum distance between parallel tangents) from the side view image and the Width (axial major axis) and Length (longitudinal major axis) from the front view image (all of these described by Russ (1995)). The Minimum Feret Diameter was thereafter used for the classification of each barley kernel in one of the studied size classes. Another 12 morphological parameters were also determined: Area, Solidity, Convexity, Aspect Ratio, Compactness, Extent and Shape Factor (all of these described by Russ (1995)); Roundness (Glasbey and Horgan, 1994); Robustness (Pons et al., 1997); morphological parameters Par $A$ and Par B, and a Kernel Volume Descriptor (KVD) were determined as follows:

$$
\begin{aligned}
\operatorname{ParA} & =\frac{\operatorname{abs}\left(X_{\mathrm{M}}^{\mathrm{C}}-X_{\mathrm{BB}}^{\mathrm{C}}\right)+\operatorname{abs}\left(Y_{\mathrm{M}}^{\mathrm{C}}-Y_{\mathrm{BB}}^{\mathrm{C}}\right)}{\text { Area }} \\
\operatorname{ParB} & =\frac{\max \left[\operatorname{abs}\left(X_{\mathrm{M}}^{\mathrm{C}}-X_{\mathrm{BB}}^{\mathrm{C}}\right) ; \operatorname{abs}\left(Y_{\mathrm{M}}^{\mathrm{C}}-Y_{\mathrm{BB}}^{\mathrm{C}}\right)\right]}{\text { Area }} \\
\mathrm{KVD} & =(\text { Width } \times \text { Length } \times \text { Height })
\end{aligned}
$$

where $X_{M}^{C}$ and $Y_{M}^{C}$ are the $X$ and $Y$ mass centers, respectively, and $X_{\mathrm{BB}}^{\mathrm{C}}$ and $Y_{\mathrm{BB}}^{\mathrm{C}}$ are the bounding box $X$ and $Y$ centers, respectively.

The third stage encompassed the determination of the camera minimum acceptable resolution and the minimum number of kernels to be acquired. The effect of the proposed camera resolution, for an estimated error of one pixel in the determination of the kernel minor axial axis, was studied regarding its propagation towards the barley weight percentages assessment. Thus, in the current work, weight percentages were determined, for each barley variety and size class, for the obtained kernel minor axial axis, and for under and overestimating the minor axial axis by one pixel, and their influence established. With respect to the kernel sampling size a study was performed comparing the weight percentages assessment obtained by the overall set of images (validation and training sets representing 585 kernels), and by the validation set (245 kernels) for each barley variety and size class.

The fourth stage consisted on developing the PLS model to estimate the kernel weight of the barley from the IA morphological data. With that purpose a training set of 7 images in average (corresponding to around 340 kernels) for each barley variety was employed as follows. For each class of barley the kernel average weight was determined and used as the $Y$ variable of a PLS analysis applied to the IA morphological data ( $X$ variables) of the training set. In this manner, a PLS predictive model for the kernel average weight, based on the IA morphological data could be obtained.

The last stage consisted on the validation of the PLS predictive model with a validation set of 5 images (corresponding to 245 kernels) for each barley variety. Therefore, the PLS model was applied to the IA morphological data on the validation set to predict the IA based kernel weight assessment. The IA based weight distribution was then determined, by multiplying each class kernel average weight by the corresponding number percentage and, finally, the IA based and EBC standard weight distributions were plotted against each other and their correlation was examined.

\section{Results}

This methodology was developed for analysis in the field where the establishment of a robust acquisition procedure and determination of the sampling size of kernels is of the utmost importance. Therefore, emphasis was placed on camera resolution by determining the minimum acceptable resolution and the minimum number of kernels to be acquired.

Regarding the camera resolution of $1600 \times 1280$ pixels (with an average kernel region of interest of $120 \times 50$ and $120 \times 45$ pixels in 

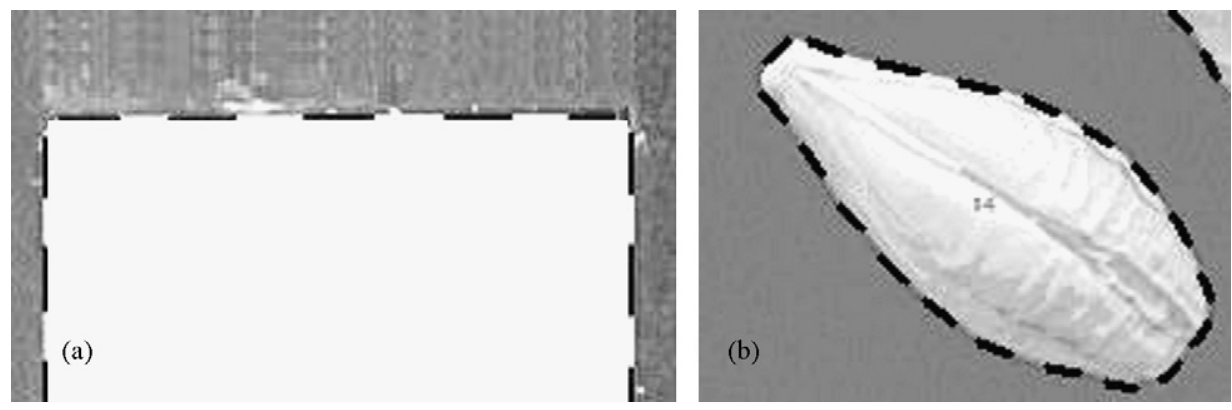

Fig. 3. Result boundaries image (a) thumbnail of the gauge block boundary (b) thumbnail of the barley kernel boundaries.

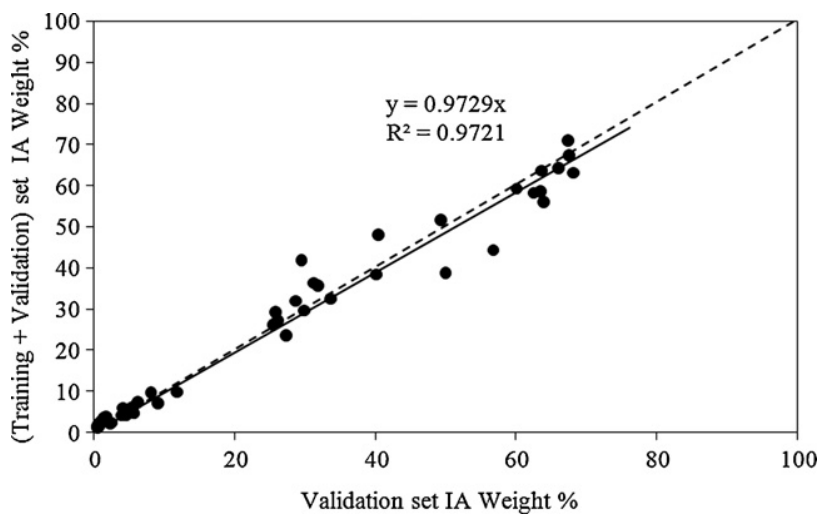

Fig. 4. Overall correlation between the IA weight distribution for the overall set of images (validation and training sets representing 585 kernels) and for the validation set (245 kernels).

the front and side views, respectively) the estimated error of one pixel $(0.056 \mathrm{~mm})$ in the determination of the minor axial axis of the kernel represented an error around $2.5 \%$. However, this error in the determination of the minor axial axis can propagate into larger errors in the determination of the barley weight percentages. As a matter of fact, during this work it was found that the weight percentage errors attained a maximum value of $6.4 \%$. However, and given the fact that the average value of all the barley weight percentage errors was of $2 \%$, the proposed camera resolution can be considered as acceptable.

The analysis of sampling size revealed that for the training set of 245 kernels ( 5 images) the results were still rather similar with a correlation factor of 0.973 and a regression coefficient of $0.986\left(R^{2}\right.$ of 0.9721 ) to the whole set analysis (585 kernels from 12 images) as it can be seen in Fig. 4 and Table 1. The weight distribution differences between the whole set and the validation set was shown to differ $1.09 \%$ on average (for the $<2.2,2.2-2.5$ and $>2.5$ classes) but, most important, for the critical below $2.2 \mathrm{~mm}$ and above $2.5 \mathrm{~mm}$ classes the differences were only of $1.09 \%$ and $1.2 \%$. These results show, thus, that no significant loss is caused by working with a 5 images sample instead of 12 images samples.

Table 1

Absolute differences between the IA weight distribution for the overall set of images (validation and training sets representing 585 kernels) and for the validation set (245 kernels) for the Esterel, Scarlett, Nevada, and Prestige barley varieties and for each size class.

\begin{tabular}{llllll}
\hline & \multicolumn{3}{l}{ Class $(\mathrm{mm})$} & & \\
\cline { 2 - 6 } & $<2.2$ & $2.2-2.5$ & $2.5-2.8$ & $>2.8$ & $>2.5$ \\
\hline Esterel & 1.87 & 1.86 & 7.24 & 8.64 & 1.41 \\
Scarlett & 1.51 & 0.55 & 1.64 & 1.97 & 1.34 \\
Nevada & 0.83 & 0.80 & 4.23 & 5.45 & 1.36 \\
Prestige & 0.13 & 0.68 & 1.70 & 2.13 & 0.70 \\
Average & 1.09 & 0.97 & 3.70 & 4.55 & 1.20 \\
\hline
\end{tabular}

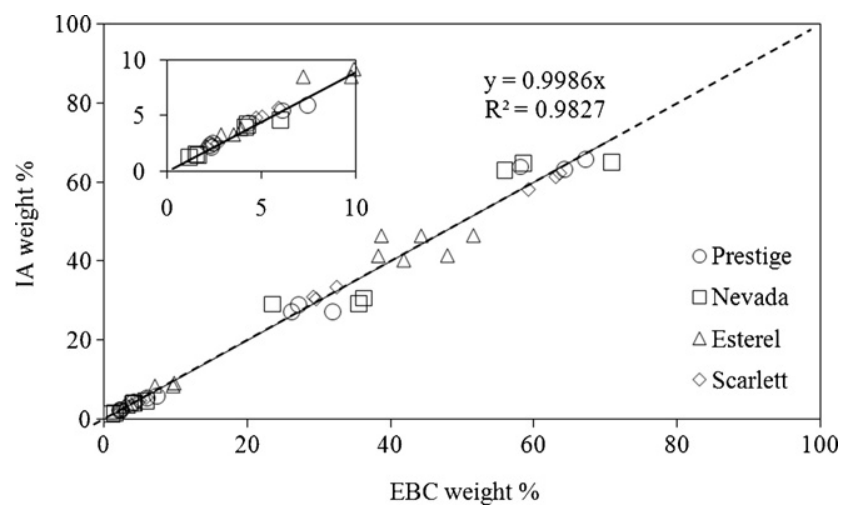

Fig. 5. Overall correlation between the IA and EBC weight percentages for the validation set (245 kernels).

Keeping in mind the previous results, an analysis between the IA and the standard EBC methodologies was subsequently performed by multivariable PLS on the 245 kernels validation set. A close correlation between the EBC weight distribution and the IA based weight distribution was obtained with a high 0.999 correlation factor and 0.991 regression coefficient ( $R^{2}$ of 0.9827 ), as it can be seen in Fig. 5 . Furthermore, the weight distribution differences between the two methodologies was shown to differ $0.49 \%$ on average (for the $<2.2$, 2.2-2.5 and $>2.5$ classes) but, most important, for the critical below $2.2 \mathrm{~mm}$ and above $2.5 \mathrm{~mm}$ classes the differences were only of $0.16 \%$ and $0.71 \%$. These results show that the time (around $10 \mathrm{~min}$ ) and effort for obtaining a total of 5 images ( 245 kernels) in the field is feasible with a simple and fast method to evaluate the barley kernel weight distribution. For each variety of barley, a correlation analysis was performed and is presented in Fig. 6. In addition, an analysis on the weight distribution differences of the barley is provided in Table 2.

Analyzing the results it is expected that the weight distribution of the Scarlett and Prestige barley varieties be accurately determined by the proposed IA methodology especially considering the greater than $2.5 \mathrm{~mm}$ class $(0.24 \%$ and $0.68 \%$ differences $)$ and less than $2.2 \mathrm{~mm}$ class $(0.11 \%$ and $0.16 \%$ differences) classes which are crucial to the supply chain management. Furthermore, the

Table 2

Absolute differences (in percentage units) between IA and EBC weight distributions for the Esterel, Scarlett, Nevada, and Prestige barley varieties and for each size class.

\begin{tabular}{llllll}
\hline & \multicolumn{5}{l}{ Class $(\mathrm{mm})$} \\
\cline { 2 - 6 } & $<2.2$ & $2.2-2.5$ & $2.5-2.8$ & $>2.8$ & $>2.5$ \\
\hline Esterel & 0.26 & 1.07 & 3.68 & 5.01 & 1.32 \\
Scarlett & 0.11 & 0.14 & 1.32 & 1.24 & 0.24 \\
Nevada & 0.10 & 0.52 & 5.82 & 6.42 & 0.60 \\
Prestige & 0.16 & 0.72 & 2.52 & 2.77 & 0.68 \\
Average & 0.16 & 0.61 & 3.34 & 3.86 & 0.71 \\
\hline
\end{tabular}



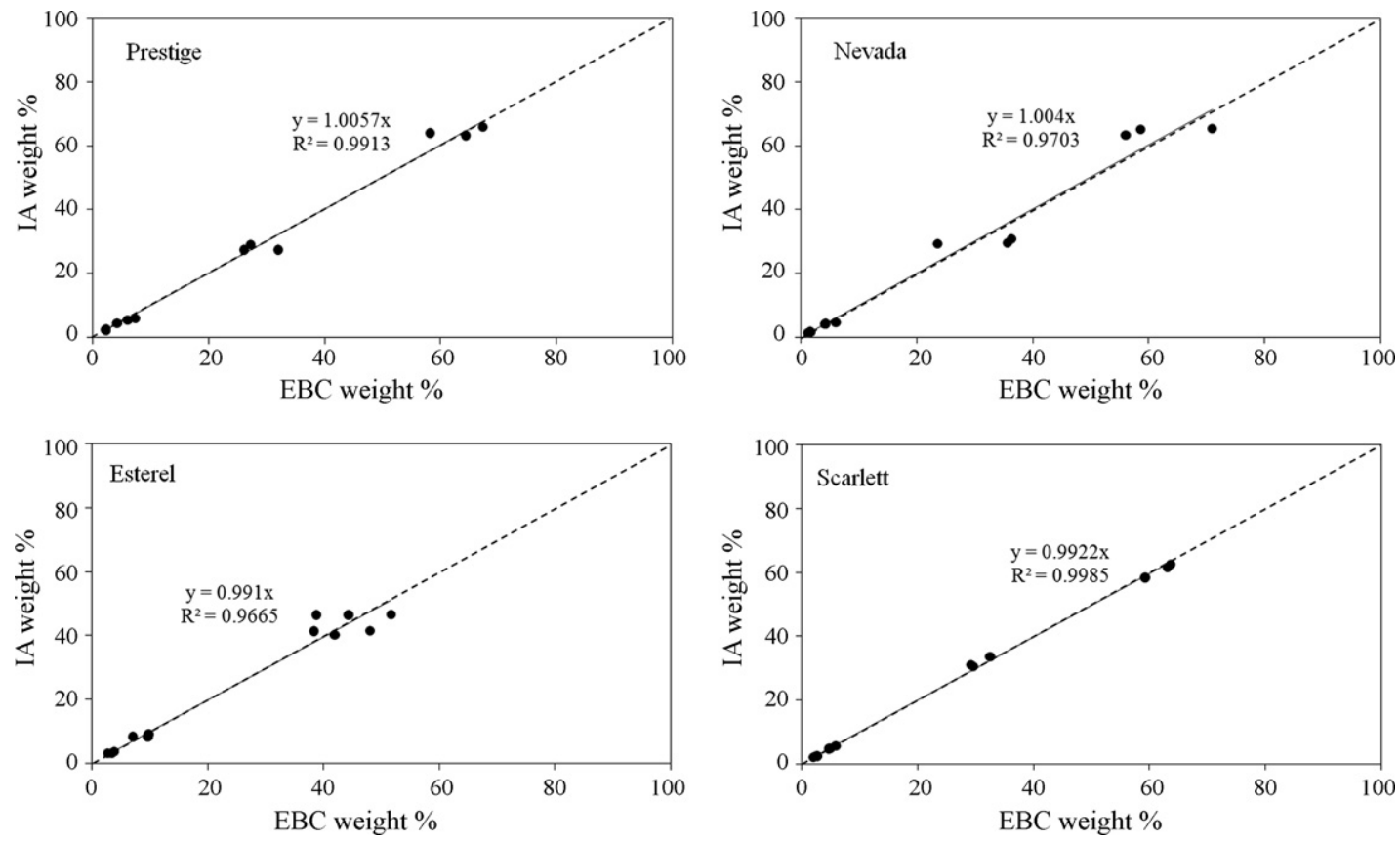

Fig. 6. Correlation between the IA and EBC weight percentages, for the Prestige, Nevada, Esterel and Scarlett barleys.

obtained correlations (0.992 and 1.006) and the high regression coefficients (above 0.99) confirmed the high correlations between both methods. However, caution is advisable concerning the weight distribution assessment within the greater than $2.5 \mathrm{~mm}$ class itself (differences up to 2.77\%). For the Esterel and Nevada barley varieties and despite good correlations were obtained (0.991 and 1.004 respectively), the results were not as satisfactory due to the lower regression coefficients of 0.983 and 0.985 respectively $\left(R^{2}\right.$ of 0.9665 and 0.9703). Although the results found for the Esterel and Nevada barley varieties, regarding the greater than $2.5 \mathrm{~mm}$ (1.32\% and $0.60 \%$ differences) and less than $2.2 \mathrm{~mm}$ (0.26\% and $0.10 \%$ differences) classes were satisfactory, and especially regarding the Nevada bar- ley, the low number of data points available (12) may be hindering its reliability for the time being. Thus, only a more in-depth analysis with future field work values can provide more reliable results in order to validate the effectiveness of this method regarding these barleys.

The main morphological parameters from the Partial Least Squares analysis (obtained for a maximum of 6 PLS components), governing the weight dependence, are presented in Fig. 7, in terms of their variable importance. The average variable importance of the studied parameters for the different types of barley reveals that the most significant were the Extent (1.203), Shape Factor (1.196), Convexity (1.132), Solidity (1.130), Par B (1.118) and Length (1.070).
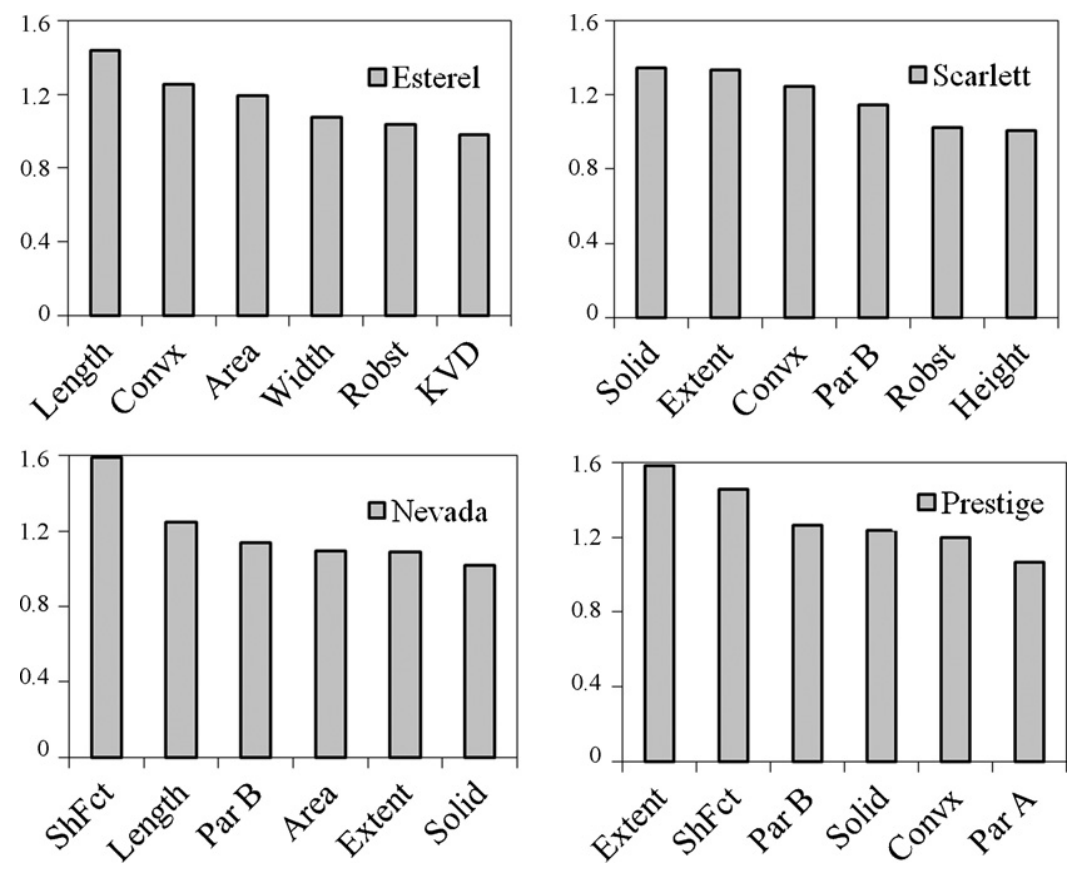

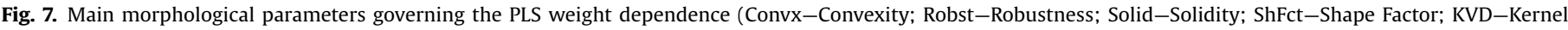
Volume Descriptor; Par A-Morphological Parameter A defined in Eq. (1); Par B-Morphological Parameter B defined in Eq. (2)). 
However it was also found a great heterogeneity regarding the most important parameters for each barley variety weight prediction. In fact, 12 different parameters presented a variable importance higher than 1 in at least one of the barley varieties. This shows that for each barley variety specific morphological descriptors must be determined representing the complexity in obtaining an overall model for the estimation of the IA based barley weight distribution.

\section{Discussion}

During this study it was determined that the accuracy of the proposed IA method weight distribution was somewhat sensitive to the image resolution although, and given the $2 \%$ weight percentage error average, a $1600 \times 1280$ pixels camera resolution can be considered as acceptable. Furthermore, it was also found that a simple and fast collection of a total of 5 images ( 245 kernels), was sufficient to obtain an overall good prediction ability ( 0.999 correlation factor with 0.991 regression coefficient) of the barleys weight distribution by the current methodology.

The results of the PLS analysis between the IA and the standard EBC methodologies on the 245 kernels validation set revealed a close correlation between both methods. The obtained correlation factor of 0.999 and regression coefficient of 0.991 between the two methodologies could be considered quite satisfactory. It was also found that the proposed image analysis methodology can be expected to accurately predict the Scarlett and Prestige barley varieties weight distribution, especially when considering the crucial 'business transactions selection' classes. However, further studies need to be made in order to obtain a more reliable kernel weight distribution by the proposed IA methodology for the Esterel and Nevada barley varieties.

Furthermore, the heterogeneity regarding the most important parameters for each barley variety weight prediction also revealed the complexity in obtaining an overall model for the estimation of the IA based barley weight distribution.

The results obtained in this study open many possibilities for the implementation of this simple, fast, and affordable method for field supply chain management. Indeed, progress is already being made to adapt this methodology with minor changes.

\section{Conclusions}

The developed methodology allows for the implementation of a simple, fast, and affordable image analysis procedure for barley kernel size evaluation in the field. This methodology facilitates the barley purchasing process, as a fast and simple procedure for barley selection, and allows the implementation of field supply chain management.

\section{Acknowledgements}

The authors are grateful to Unicer, S.A. for their financial and material support throughout this work. Special thanks are due to Dr. Anthony S. Danko for his helpful comments and corrections.

\section{References}

Amaral, A.L., Rocha, O., Gonçalves, C., Ferreira, A.A., Ferreira, E.C., 2005. Development of image analysis methods to evaluate barley/malt grain size. In: Proceedings of the CHEMPOR 2005-9th International Chemical Engineering Conference, Coimbra, Portugal, CD-ROM.

Analytica EBC, 1998. Sieving Test for Barley Method 3.11.

Andersson, A.A.M., Elfverson, C., Andersson, R., Regnér, S., Åman, P., 1999. Chemical and physical characteristics of different barley samples. J. Sci. Food Agric. 79, 979-986.

Brennan, C.S., Harris, N., Smith, D., Shewry, P.R., 1996. Structural differences in the mature endosperms of good and poor malting barley cultivars. J. Cereal Sci. 24, $171-177$.

Brosnan, T., Sun, D.W., 2004. Improving quality inspection of food products by computer vision-a review. J. Food Eng. 61, 3-16.

Dougherty, E.R., 1994. Digital Image Processing Methods. Marcel Dekker Inc., New York.

Edney, M.J., Rossnagel, B.G., Endo, Y., Ozawa, S., Brophyk, M., 2002. Pearling quality of canadian barley varieties and their potential use as rice extenders. J. Cereal Sci. 36, 295-305.

Garciǐa del Moral, L.F., Sopena, A., Montoya, J.L., Polo, P., Voltas, J., Codesal, P., Ramos, J.M., Molina-Cano, J.L., 1998. Image analysis of grain and chemical composition of the barley plant as predictors of malting quality in Mediterranean environments. Cereal Chem. 75, 755-761.

Glasbey, C.A., Horgan, G.W., 1994. Image Analysis for the Biological Sciences. John Wiley \& Sons, New York.

Gonzalez, R.C., Woods, R.E., 1992. Digital Image Processing. Addison-Wesley Publishing Company, Reading.

Majumdar, S., Jayass, D.S., 1999. Classification of bulk samples of cereal grains using machine vision. J. Agric. Eng. Res. 73, 35-47.

Nielsen, J.P., 2003. Evaluation of malting barley quality using exploratory data analysis II. The use of kernel hardness and image analysis as screening methods. J. Cereal Sci. 38, 247-255.

Otsu, N., 1979. A threshold selection method from gray-level histograms. IEEE Trans. Syst. Man Cybern. 1, 62-66.

Pons, M.N., Vivier, H., Dodds, J., 1997. Particle shape characterization using morphological descriptors. Part. Syst. Charact. 14, 272-277.

Russ, C.R., 1995. The Image Processing Handbook. CRC Press, Boca Raton.

Shouche, S.P., Rastogi, R., Bhagwat, S.G., Sainis, J.K., 2001. Shape analysis of grains of Indian wheat varieties. Comp. Electron. Agric. 33, 55-76.

Utku, H., 2000. Application of the feature selection method to discriminate digitized wheat varieties. J. Food Eng. 46, 211-216. 